\title{
The relationship between lung function impairment and quantitative computed tomography in chronic obstructive pulmonary disease
}

\author{
O. M. Mets • K. Murphy • P. Zanen • H. A. Gietema • \\ J. W. Lammers • B. van Ginneken • M. Prokop • \\ P. A. de Jong
}

Received: 5 April 2011 /Revised: 1 June 2011 / Accepted: 2 July 2011 /Published online: 12 August 2011

(C) The Author(s) 2011. This article is published with open access at Springerlink.com

\begin{abstract}
Objectives To determine the relationship between lung function impairment and quantitative computed tomography (CT) measurements of air trapping and emphysema in
\end{abstract}

O. M. Mets $\cdot$ H. A. Gietema $\cdot$ M. Prokop $\cdot$ P. A. de Jong

Radiology, University Medical Center Utrecht,

Heidelberglaan 100,

3508GA, Postbus 85500, Utrecht, The Netherlands

K. Murphy $\cdot$ B. van Ginneken

Image Sciences Institute, University Medical Center Utrecht,

Heidelberglaan 100

3508GA, Postbus 85500, Utrecht, The Netherlands

P. Zanen $\cdot$ J. W. Lammers

Pulmonology, University Medical Center Utrecht, Utrecht,

The Netherlands,

Heidelberglaan 100 ,

3508GA, Postbus 85500, Utrecht, The Netherlands

B. van Ginneken

Diagnostic Image Analysis Group, Radiology,

Radboud University Nijmegen Medical Centre,

Anthonie van Leeuwenhoeklaan 5,

6525 HK, Nijmegen, The Netherlands

M. Prokop

Radiology, Radboud University Nijmegen Medical Centre,

The Netherlands,

Geert Grooteplein-Zuid 10,

6525 GA, Nijmegen, The Netherlands

O. M. Mets $(\bowtie)$

Department of Radiology, University Medical Center Utrecht,

Huispostnummer E01.132, Postbus 85500, 3508 GA, Utrecht,

The Netherlands

e-mail: o.m.mets@umcutrecht.nl a population of current and former heavy smokers with and without airflow limitation.

Methods In 248 subjects (50 normal smokers; 50 mild obstruction; 50 moderate obstruction; 50 severe obstruction; 48 very severe obstruction) CT emphysema and CT air trapping were quantified on paired inspiratory and endexpiratory CT examinations using several available quantification methods. CT measurements were related to lung function $\left(\mathrm{FEV}_{1}, \mathrm{FEV}_{1} / \mathrm{FVC}, \mathrm{RV} / \mathrm{TLC}, \mathrm{Kco}\right)$ by univariate and multivariate linear regression analysis.

Results Quantitative CT measurements of emphysema and air trapping were strongly correlated to airflow limitation (univariate r-squared up to $0.72, p<0.001$ ). In multivariate analysis, the combination of $\mathrm{CT}$ emphysema and $\mathrm{CT}$ air trapping explained $68-83 \%$ of the variability in airflow limitation in subjects covering the total range of airflow limitation $(p<0.001)$.

Conclusions The combination of quantitative CT air trapping and emphysema measurements is strongly associated with lung function impairment in current and former heavy smokers with a wide range of airflow limitation.

Key Points

- CT helps to automatically assess lung disease in heavy smokers

- CT quantitatively measures emphysema and small airways disease in heavy smokers

- CT air trapping and CT emphysema are associated with lung function impairment

Keywords Pulmonary emphysema $\cdot$ Respiratory bronchioles - Quantitative computed tomography . Pulmonary function test . Chronic obstructive pulmonary disease 


\section{Introduction}

Chronic Obstructive Pulmonary Disease (COPD) is characterized by chronic airflow limitation, and its severity is graded according to the Global Initiative for Chronic Obstructive Lung Disease (GOLD) criteria [1]. The airflow limitation in COPD patients is caused by parenchymal destruction (i.e. emphysema) and small airways disease with airway inflammation and airway remodeling, which leads to expiratory airway obstruction and air trapping $[2,3]$. Emphysema has been quantified in vivo by computed tomography (CT) for decades [4], but in vivo detection of small airways disease has been difficult since these small airways (diameter $<2 \mathrm{~mm}$ ) can not be visualized directly with current $\mathrm{CT}$ techniques. Nakano et al suggested that measurements of large airway wall thickening can be used as an indirect sign of small airways disease [5]. However, quantification of airway wall dimensions remains technically challenging. Alternatively, air trapping on end-expiratory CT may be easier to quantify, and is possibly a more direct measurement of small airways dysfunction. For this, the main challenge is to separate air trapping due to small airways disease from air that remains in emphysematous spaces after expiration [6].

Separate quantification of air trapping and emphysema is relevant for two reasons. First, COPD is a disease with a wide heterogeneity [7-10], and COPD phenotyping may be possible when quantitative $\mathrm{CT}$ is combined with clinical and physiological characteristics. Second, quantitative CT of COPD components might gain a role in (early) diagnosis of the disease; a risk estimate for COPD may be calculated from CT data, providing additional information in subjects who undergo CT. This strategy may be especially interesting in lung cancer screening, where large numbers of CT examinations are obtained in heavy smokers. Here, CT may reveal 'hidden' disease in undiagnosed COPD patients, and might reveal disease in patients with lung function still within the normal range [11].

The purpose of this study was to determine the relationship between lung function impairment and quantitative CT measures of air trapping and emphysema in heavy smokers with and without COPD.

\section{Methods}

To study COPD in a lung cancer screening trial [12], pulmonary function tests (PFT) and an expiratory CT data acquisition had previously been added to the screening protocol in our center. In the present study, we automatically assessed emphysema and air trapping in CT examinations obtained in this lung cancer screening population.
Since limited subjects with severe airflow limitation were present in the screening trial, we added clinical subjects to be able to study the total range of COPD.

This retrospective study was approved by our institutional ethics board; written informed consent was obtained in the screening trial participants, and waived for the clinical subjects.

\section{Subjects}

Within the lung cancer screening trial participants, random samples of 50 subjects were drawn for each GOLD stage [1], resulting in 50 GOLD 1, 50 GOLD 2 and 24 GOLD 3 subjects. The clinical subjects underwent outpatient inspiratory and expiratory CT and PFT examination for evaluation of COPD and/or lung transplantation screening. Cases with GOLD 3 of GOLD 4 were selected. No CTs contained major breathing artifacts, and scans with signs of acute pneumonia were excluded. The GOLD 3 study population was extended to 50 subjects with a random sample of clinical subjects. The GOLD 4 group consisted of all available clinical subjects $(N=48)$.

Additionally, we selected a group of normal smokers from the screening trial participants. We defined 'normal smoker' as two normal lung function tests with at least a two-year interval, plus a slow rate of lung function decline. Normal lung function was defined as $\mathrm{FEV}_{1}$ (forced expiratory volume in one second) $>80 \%$ predicted and $\mathrm{FEV}_{1} / \mathrm{FVC}$ (forced expiratory volume in one second / forced vital capacity) $>70 \%$. A slow rate of decline was defined as an $\mathrm{FEV}_{1}$ decline less than $29 \mathrm{ml}$ per year [13]. Seventy subjects fulfilled these criteria, of whom 50 were randomly selected.

\section{Pulmonary function testing}

Spirometry, body plethysmography and diffusion capacity testing was obtained according to ERS guidelines [14]. PFT was performed with ZAN, Oberthulba, Germany. Spirometry was obtained in all subjects and included forced expiratory volume in 1 second $\left(\mathrm{FEV}_{1)}\right.$, forced vital capacity (FVC) and the ratio of forced expiratory volume in 1 second over forced vital capacity $\left(\mathrm{FEV}_{1} / \mathrm{FVC}\right)$. Data on bronchodilator use on the day of examination was not available, and reversibility with bronchodilation was not assessed. Due to restrictions in testing capacity during screening rounds, extensive pulmonary function testing was only performed in a subgroup of subjects. For our study population, RV/TLC (ratio of residual volume to total lung capacity) was available in 121 subjects, and Kco (ratio of diffusion capacity over alveolar volume) in 103 subjects. For lung cancer screening subjects, PFT was performed on the same day as CT. For clinical subjects, the PFT 
performed closest to the $\mathrm{CT}$ date was selected (median 1 day, interquartile range $0-4$ days).

\section{Computed tomography}

Volumetric inspiratory $\mathrm{CT}$ and volumetric end-expiratory $\mathrm{CT}$ after standardized breathing instructions were obtained between July 2007 and September 2008. All CT examinations were performed without intravenous contrast medium, using 16 slice CT (Brilliance 16P, Philips Medical Systems, Cleveland, $\mathrm{OH}$ ) and $0.75 \mathrm{~mm}$ collimation. Settings were adjusted to body weight: $120 \mathrm{kVp}(\leq 80 \mathrm{~kg})$ or $140 \mathrm{kVp}$ $(>80 \mathrm{~kg}$ ), both with $30 \mathrm{mAs}$, for inspiratory CT, and $90 \mathrm{kVp}$ $(\leq 80 \mathrm{~kg})$ or $120 \mathrm{kVp}(>80 \mathrm{~kg})$, both with $20 \mathrm{mAs}$, for expiratory CT. A smooth reconstruction filter (B filter, Philips, Cleveland, $\mathrm{OH}$ ) was used, according to the protocol. Images with slice thickness of $1.0 \mathrm{~mm}$ at $0.7 \mathrm{~mm}$ increment were reconstructed from lung bases to lung apices.

The clinically evaluated patients that were added to the screening population, underwent volumetric inspiratory CT and volumetric end-expiratory $\mathrm{CT}$ after standardized breathing instructions between October 2003 and May 2010. CT was performed without any intravenous contrast medium. These CT examinations were acquired on various generations of $\mathrm{CT}$ equipment from the same vendor (Philips Medical Systems, Cleveland, $\mathrm{OH})$ with $16 \times 0.75 \mathrm{~mm}$ collimation on Brilliance 16P $(N=16)$ and $\mathrm{Mx} 8000$ IDT $(N=12), 40 \times 0.625 \mathrm{~mm}$ collimation on Brilliance $40(N=8)$, $64 \times 0.625 \mathrm{~mm}$ collimation on Brilliance $64(N=32)$ or $128 \times 0.625 \mathrm{~mm}$ collimation on Brilliance iCT $(N=6)$. Exposure settings were: $100 \mathrm{kVp}$ or $120 \mathrm{kVp}$, both with $130 \mathrm{mAs}$, for inspiratory $\mathrm{CT}$, and $80-120 \mathrm{kVp}$ with $10-$ $30 \mathrm{mAs}$ for expiratory CT. A sharp reconstruction filter (C kernel, Philips) was used, according to the clinical protocol. Images with slice-thickness of either 0.9 or $1.0 \mathrm{~mm}$ at 0.45 or $0.7 \mathrm{~mm}$ slice-spacing were reconstructed from lung bases to lung apices.

Emphysema and air trapping quantification

The lung parenchyma on inspiratory and expiratory CT data sets was automatically segmented [15]. Additionally, lung segmentation was checked visually, and those with major errors (see Appendix 1) were excluded. Subjects that were excluded were replaced by another random subject with a corresponding GOLD stage $(N=16)$. A noise reduction filter [16] was applied to decrease the influence of noise on the quantitative CT measurements.

The extent of emphysema was assessed by two widely used quantitative CT measures:

1) The percentage of lung voxels below $-950 \mathrm{HU}$ in inspiration $\left(\mathrm{IN}_{-950}\right)[17,18]$.
2) The HU value at the 15th percentile of the attenuation distribution histogram in inspiration $\left(\right.$ Perc $\left._{15}\right)$ [19].

The extent of air trapping was quantified by four different methods:

1) The percentage of lung voxels below $-850 \mathrm{HU}$ in expiration $\left(\mathrm{EXP}_{-850}\right)[20,21]$.

2) The percentage of lung voxels between $-850 \mathrm{HU}$ and $910 \mathrm{HU}$ in expiration $\left(\mathrm{EXP}_{-850}\right.$ to -910$)$. To correct for air in emphysematous areas, method 1 was adjusted by excluding the voxels below $-910 \mathrm{HU}$, based on reference [22].

3) The change in relative lung volume with attenuation values from $-860 \mathrm{HU}$ to $-950 \mathrm{HU}\left(\mathrm{RVC}_{-860}\right.$ to -950$)$ between paired inspiratory and expiratory examinations [6]. Practically, the $\mathrm{RVC}_{860-950}$ is calculated using the formula: expiratory relative lung volume -860 to $-950 \mathrm{HU}$ inspiratory relative lung volume -860 to $-950 \mathrm{HU}$. In this, the relative lung volume -860 to $-950 \mathrm{HU}$ is defined as the volume of voxels with attenuation values between -860 to $-950 \mathrm{HU}$ divided by the volume of voxels with attenuation values above $-950 \mathrm{HU}$ (i.e. limited lung volume, without emphysema) [6].

4) The expiration to inspiration ratio of mean lung density (E/I-ratio $\left.{ }_{\text {MLD }}\right)$ [23-25].

\section{Data analysis}

CT measurements were compared between the five groups (i.e. normal smokers and the four COPD stages) with analysis of covariance (ANCOVA), with age, height and sex as covariates. Univariate and multivariate linear regression analysis were performed to evaluate the relationship between lung function parameters (dependent variables) and the CT measurements (independent variables). Multivariate analysis incorporated CT emphysema (2 methods) and CT air trapping (4 methods), while age, sex and height were introduced as correction factors for PFT. In order to meet linear regression requirements, we used absolute values instead of the 'percentage of predicted value', as the latter often elicits a heteroscedastic distribution of the residuals to one side [26]. Furthermore, multicollinearity and the distribution of residuals was assessed for all models; $\mathrm{FEV}_{1}$ and $\mathrm{IN}_{-950}$ were $\log$-transformed $\left(\operatorname{logFEV} \mathrm{F}_{1}\right.$ and $\log \mathrm{IN}_{-950}$ ) as to obtain a normal distribution, and warrant symmetrical variance of the errors around zero (i.e. homoscedasticicy). Linear regression analyses were performed in the total cohort of 248 subjects, as well as in the 174 screening trial participants separately.

All statistical analyses were performed using SPSS software v15.0 (SPSS Inc, Chicago, Illinois, USA). A pvalue below 0.05 was considered statistical significant. Continuous data are given as mean $\pm \mathrm{SD}$, unless indicated otherwise. 
Table 1 Patient characteristics, pulmonary function and quantitative CT measurements

\begin{tabular}{|c|c|c|c|c|c|}
\hline & Controls $(n=50)$ & GOLD1 $(n=50)$ & GOLD2 $(n=50)$ & GOLD3 $(n=50)$ & GOLD4 $(n=48)$ \\
\hline Male, n (\%) & $50(100)$ & $48(96)$ & $49(98)$ & $37(74)$ & $16(33)$ \\
\hline Age (years) & $62.8 \pm 5.8$ & $62.6 \pm 4.8$ & $62.8 \pm 5.5$ & $62.4 \pm 11$ & $55.5 \pm 7.3$ \\
\hline \multicolumn{6}{|l|}{ Pulmonary Function } \\
\hline Days between PFT and CT, median [P25 - P75] & $0[-]$ & $0[-]$ & $0[-]$ & $0[0-3]$ & $1[0-2]$ \\
\hline $\mathrm{FEV}_{1}(\mathrm{~L})$ & $3.58 \pm 0.55$ & $3.18 \pm 0.52$ & $2.28 \pm 0.46$ & $1.26 \pm 0.31$ & $0.62 \pm 0.20$ \\
\hline FEV1 (\%predicted) & $105.4 \pm 11.5$ & $94.8 \pm 9.1$ & $68.9 \pm 9.3$ & $41.2 \pm 5.7$ & $21.7 \pm 5.1$ \\
\hline $\mathrm{FEV}_{1} / \mathrm{FVC}(\%)$ & $75.6 \pm 3.9$ & $65.3 \pm 4.1$ & $60.3 \pm 7.2$ & $42.2 \pm 7.6$ & $31.8 \pm 9.1$ \\
\hline $\mathrm{RV} / \mathrm{TLC}(\%)^{\mathrm{a}}$ & $34.6 \pm 6.7$ & $35.7 \pm 7.4$ & $47.0 \pm 9.2$ & $56.8 \pm 7.0$ & $66.0 \pm 8.2$ \\
\hline $\mathrm{Kco}(\mathrm{mmol} / \mathrm{min} / \mathrm{kPa} / \mathrm{L})^{\mathrm{b}}$ & $1.32 \pm 0.23$ & $1.12 \pm 0.26$ & $1.20 \pm 0.30$ & $0.88 \pm 0.32$ & $0.72 \pm 0.22$ \\
\hline \multicolumn{6}{|l|}{ CT Emphysema ${ }^{c}$} \\
\hline $\mathrm{IN}_{-950}(\%)$ & $1.0 \pm 0.8$ & $2.1 \pm 1.7^{*}$ & $3.4 \pm 5.2^{* \dagger}$ & $11.6 \pm 10.0$ & $27.9 \pm 14.3$ \\
\hline $\operatorname{Perc}_{15}(\mathrm{HU})$ & $-906 \pm 20$ & $-919 \pm 12$ & $-920 \pm 19^{\dagger}$ & $-938 \pm 21$ & $-960 \pm 22$ \\
\hline \multicolumn{6}{|l|}{ CT Air trapping ${ }^{\mathrm{c}}$} \\
\hline $\mathrm{EX}_{-850}(\%)$ & $11.3 \pm 9.6$ & $16.5 \pm 14.4$ & $25.9 \pm 17.9$ & $50.0 \pm 16.8$ & $70.0 \pm 12.8$ \\
\hline $\mathrm{EX}_{-850 \text { to-910 }}(\%)$ & $9.9 \pm 8.6$ & $13.8 \pm 11.0^{*}$ & $20.9 \pm 13.3$ & $31.4 \pm 9.9$ & $25.6 \pm 6.8^{\ddagger}$ \\
\hline $\mathrm{RVC}_{-860 t o-950}(\%)$ & $-54.2 \pm 18.3$ & $-60.0 \pm 13.8$ & $-49.2 \pm 16.2^{*}$ & $-23.4 \pm 13.1$ & $-6.8 \pm 6.8$ \\
\hline E/I-ratio ${ }_{M L D}(\%)$ & $84.5 \pm 4.2$ & $84.8 \pm 4.6^{*}$ & $88.4 \pm 4.5$ & $93.8 \pm 4.3$ & $97.5 \pm 1.8$ \\
\hline
\end{tabular}

${ }^{\mathrm{a}} 121$ subjects $\left(n=31,20,14,23,33\right.$, respectively) with bodyplethysmography measurements; ${ }^{\mathrm{b}} 103$ subjects $(n=30,20,15,20,18$, respectively) with diffusion capacity measurements; ${ }^{\mathrm{c}}$ All categories are significantly different (p-value between $<0.05$ and $<0.001$ ), except when stated otherwise; ${ }^{*}$ no significant difference compared to controls; ${ }^{\dagger}$ no significant difference compared to GOLD1; ${ }^{*}$ no significant difference compared to GOLD3; IQR Interquartile range; $I_{-950}=$ emphysema score below $-950 \mathrm{HU}$ in inspiratory scan; Perc $15=$ emphysema score as $15^{\text {th }}$ percentile of attenuation distribution curve in inspiratory scan; $\mathrm{EXP}_{-850}=$ air trapping score below $-850 \mathrm{HU}$ in expiratory scan; $\mathrm{EXP}_{-850 t o-910}=$ air trapping score between $-850 \mathrm{HU}$ and $-910 \mathrm{HU}$ in expiratory scan; $\mathrm{RVC}_{-860 t o-950}=$ air trapping score as relative volume change between $-860 \mathrm{HU}$ and $-950 \mathrm{HU}$; E/Iratio $_{\mathrm{MLD}}=$ expiration to inspiration ratio of mean lung density

Table 2 Results of univariate linear regression analysis for quantitative CT measurements of emphysema and air trapping and pulmonary function tests

\begin{tabular}{lcccc}
\hline & $\operatorname{logFEV}_{1}$ & $\mathrm{FEV}_{1} / \mathrm{FVC}$ & $\mathrm{RV}_{\text {TLC }}{ }^{\mathrm{a}}$ & $\mathrm{Kco}^{\mathrm{b}}$ \\
\hline CT Emphysema & & & & \\
$\operatorname{logIN}_{\text {-950 }}$ & 0.53 & 0.61 & 0.42 & 0.52 \\
Perc $_{15}$ & 0.44 & 0.53 & 0.35 & 0.45 \\
CT Air trapping & & & & \\
EX $_{-850}$ & 0.69 & 0.72 & 0.63 & 0.44 \\
EX $_{-850 \text { to-910 }}$ & 0.25 & 0.33 & 0.31 & 0.18 \\
RVC $_{-860 t o-950}$ & 0.66 & 0.58 & 0.51 & 0.27 \\
E/I-ratio $_{\text {MLD }}$ & 0.60 & 0.60 & 0.56 & 0.31 \\
\hline
\end{tabular}

Data given are r-squared $\left(\mathrm{R}^{2}\right)$ values, with $p<0.001$ for all results

${ }^{a} 121$ subjects with body box measurements; ${ }^{b} 103$ subjects with diffusion capacity measurements; $\log \mathrm{IN}_{-950}=\log$-transformed emphysema score below $-950 \mathrm{HU}$ in inspiratory scan; Perc15= emphysema score as $15^{\text {th }}$ percentile of attenuation distribution curve in inspiratory scan; $\mathrm{EXP}_{-850}=$ air trapping score below $-850 \mathrm{HU}$ in expiratory scan; $\mathrm{EXP}_{-850 \text { to-910 }}=$ air trapping score between $-850 \mathrm{HU}$ and $-910 \mathrm{HU}$ in expiratory scan; $\mathrm{RVC}_{-860 \text { to- } 950}=$ air trapping score as relative volume change between $-860 \mathrm{HU}$ and $-950 \mathrm{HU} ; \mathrm{E} / \mathrm{I}-$ ratio $_{\mathrm{MLD}}=$ expiration to inspiration ratio of mean lung density

\section{Results}

Study population characteristics

The majority were male and the average age was around 60 years, except for the subjects in the GOLD 4 group who were younger and more often female (Table 1). CT emphysema and $\mathrm{CT}$ air trapping results were worse in the COPD cases when compared to the smokers with normal lung function, and the results worsened in the subsequent COPD stages. Differences were significant except for $\mathrm{IN}_{-950}$ between normal smokers to GOLD2, Perc15 between GOLD1 and GOLD2, EXP -850 to -910 and E/I-ratio ${ }_{\text {MLD }}$ between normal smokers and GOLD1, $\mathrm{RVC}_{-860}$ to -950 between normal smokers and GOLD2, and $\mathrm{EXP}_{-850}$ to -910 between GOLD3 and GOLD4.

Univariate regression analysis

Quantitative CT measurements showed moderate to strong association with lung function parameters of airflow limitation (R-squared values ranging from 0.25 to $0.72, p$ $<0.001$ (Table 2, Fig. 1). CT emphysema measurements were better correlated to Kco compared to RV/TLC. Also, $\mathrm{CT}$ air trapping measurements were better correlated to RV/ 

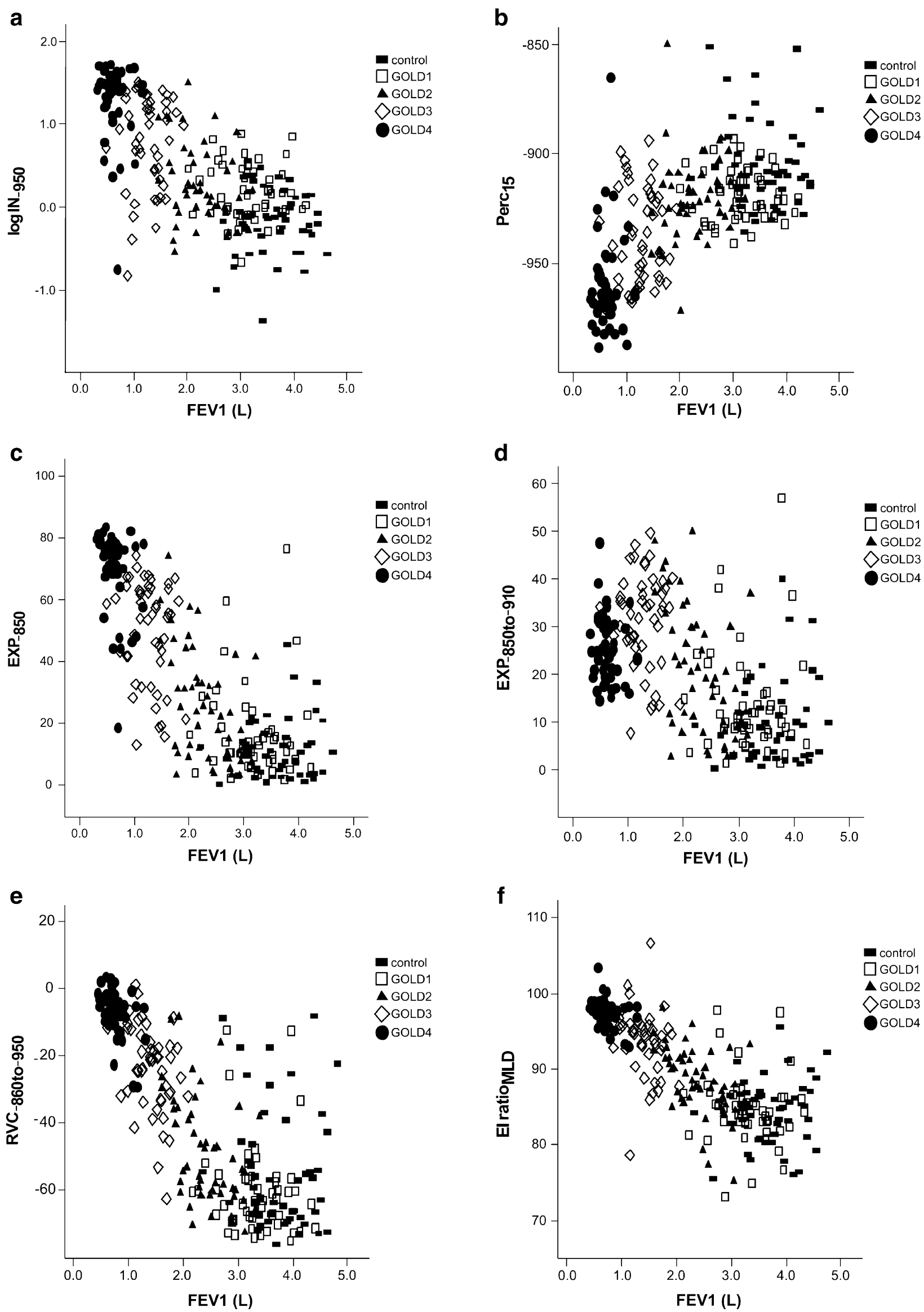
Fig. 1 Graphical illustration of the relationship between quantitative CT measurements and airflow obstruction (FEV1). The relationship between the quantitative $\mathrm{CT}$ measures and the forced expiratory volume in one second (FEV1). A log-transformed emphysema score below $-950 \mathrm{HU}$ in inspiratory scan $(\operatorname{logIN}-950)$ versus FEV1; B emphysema score as 15 th percentile of attenuation distribution curve in inspiratory scan (Perc15) versus FEV1; C air trapping score below $850 \mathrm{HU}$ in expiratory scan (EXP-850) versus FEV1; D air trapping score between $-850 \mathrm{HU}$ and $-910 \mathrm{HU}$ in expiratory scan (EXP-850 to 910) versus FEV1; E air trapping score as relative volume change between -860 HUand $-950 \mathrm{HU}$ (RVC-860 to -950 ) versus FEV1; F expiration to inspiration ratio of mean lung density (E/I-ratioMLD) versus FEV1; GOLD global initiative for chronic obstructive lung disease

TLC compared to Kco. These findings did not change when only the screening trial participants were included, though the associations were weaker after exclusion of the more severely affected subjects (Appendix 2).

\section{Multivariate regression analysis}

Testing of collinearity and residuals showed a variance inflation factor below 10 [27] in all models (max: 3.85), and a normal, homoscedastic distribution of residuals. The multivariate CT models largely explained the variability in airflow limitation; R-squared value 0.68 to 0.83 (Table 3). This pattern did not change when only the screening trial participants were included, though the explained variance was lower after exclusion of the more severely affected subjects; R-squared value 0.49 to 0.59 (Table 3 ).

\section{Discussion}

In a population that covered the range of airflow limitation from mild to very severe obstruction, we showed that the combination of quantitative CT measurements of emphysema and air trapping is strongly associated with lung function impairment, and explains a large proportion of the variability in airflow limitation.

Previous studies have addressed the small airways disease component in COPD by quantitative CT, but used large airway wall dimensions as a surrogate for small airways disease [28]. Nakano et al reported on the combination of large airway wall thickening and quantitative emphysema to explain lung function variability and to detect COPD subgroups. They evaluated 114 smokers, and showed that quantitative CT measurements of emphysema and airways wall thickness explained $23 \%$ to $49 \%$ of lung function variability [28]. In the present study we found a higher R-squared value; $68 \%$ to $83 \%$ for the whole study population, and $49 \%$ to $58 \%$ in the screening participants only. This difference in explained variance compared to the study of Nakano et al., who found an R-squared of $23 \%$ to $49 \%$, is likely due to the fact that air trapping is a more direct measure of small airways dysfunction compared to airway wall thickness. Additionally, part of the increase may be due to differences between the study populations. Previously, Nakano et al showed that airway wall thickening in CT closely correlates with small airway dimensions in histological specimens, and thus can be used as an indirect sign of small airways disease [5]. Other groups have shown that the correlation between large airway measurements and lung function parameters increases with subsequent airway generations [29-32]. This supports our view that the signal is strongest in the more peripheral airways. Additionally, quantification of airway wall dimensions remains technically challenging, especially in the more distal generations (i.e. beyond the fifth generation). Therefore, assessment of air trapping with quantitative

Table 3 Results of multivariate linear regression for quantitative CT measurements of emphysema and air trapping and pulmonary function tests

\begin{tabular}{|c|c|c|c|c|}
\hline \multirow[b]{2}{*}{ CT Model } & \multicolumn{2}{|c|}{ All subjects $(N=248)$} & \multicolumn{2}{|c|}{ Subanalysis $(N=174)^{\text {a }}$} \\
\hline & $\operatorname{logFEV} 1$ & FEV1/FVC & $\operatorname{logFEV} 1$ & FEV1/FVC \\
\hline $\log \mathrm{IN}_{-950}+\mathrm{EXP}_{-850}$ & 0.81 & 0.76 & 0.54 & 0.58 \\
\hline $\log I \mathrm{~N}_{-950}+\mathrm{EXP}_{-850 \text { to- } 910}$ & 0.75 & 0.72 & 0.51 & 0.56 \\
\hline $\log \mathrm{IN}_{-950}+\mathrm{RVC}_{-860 t o-950}$ & 0.83 & 0.75 & 0.53 & 0.55 \\
\hline $\log \mathrm{IN}_{-950}+\mathrm{E} / \mathrm{I}-$ ratio $_{\mathrm{MLD}}$ & 0.81 & 0.76 & 0.54 & 0.57 \\
\hline Perc15 +EXP -850 & 0.81 & 0.75 & 0.54 & 0.55 \\
\hline Perc15 +EXP-850to-910 & 0.71 & 0.68 & 0.49 & 0.49 \\
\hline Perc15 +RVC -860 to-950 & 0.83 & 0.75 & 0.55 & 0.56 \\
\hline Perc15 +E/I-ratio ${ }_{M L D}$ & 0.79 & 0.73 & 0.51 & 0.51 \\
\hline
\end{tabular}

Data given are r-squared $\left(\mathrm{R}^{2}\right)$ values

${ }^{a}$ Lung cancer screening trial participants only, controls to GOLD III; $\log \mathrm{IN}_{-950}=\log$-transformed emphysema score below $-950 \mathrm{HU}$ in inspiratory scan; Perc15 $=$ emphysema score as $15^{\text {th }}$ percentile of attenuation distribution curve in inspiratory scan; EXP $-850=$ air trapping score below $850 \mathrm{HU}$ in expiratory scan; $\mathrm{EXP}_{-850 \text { to-910 }}=$ air trapping score between $-850 \mathrm{HU}$ and $-910 \mathrm{HU}$ in expiratory scan; $\mathrm{RVC}_{-860 t o-950}=$ air trapping score as relative volume change between $-860 \mathrm{HU}$ and $-950 \mathrm{HU}$; E/I-ratio ${ }_{\mathrm{MLD}}=$ expiration to inspiration ratio of mean lung density 
measures in expiratory CT may also have important technical benefits. Although there is an increase in radiation dose for an additional expiratory CT acquisition, this is limited; in our screening trial protocol the effective radiation dose was less than $0.65 \mathrm{mSv}$ for the additional expiratory CT acquisition.

Previously, the univariate relationship between quantitative CT measures and lung function in COPD is reported in a few studies [23-25, 33, 34]. In these studies, correlation coefficients between E/I-ratio ${ }_{M L D}$ and $\mathrm{FEV}_{1}$ of 0.45 and $0.64(p<$ 0.001 ) are reported, which is lower than what we found. Reported correlation coefficients with RV/TLC are between 0.54 and 0.71 , which is also slightly lower than our results. These differences may well be due to differences in study populations, since our study population was larger and equally covered all the stages of COPD. Further, $\mathrm{RVC}_{-860}$ to -950 has been previously studied by Matsuoka and colleagues in a cohort of 36 COPD subjects with GOLD stage 1-4 [6]. They found correlation coefficients of 0.80 and 0.78 between this CT air trapping measure and $\mathrm{FEV}_{1}$ and $\mathrm{FEV}_{1} / \mathrm{FVC}$, respectively [6]. Despite the considerably smaller study population their results are comparable to ours, both for the whole population, and the less severely affected trial participants only. Last, the $\mathrm{EXP}_{-850}$ method has been published only in abstract format [20], and was previously used in asthma research for quantification of air trapping [21].

One has to be careful with the conclusion that one method is better than the other, given the absence of a clear reference standard for either emphysema or air trapping in our study. In fact, the main purpose of our study was to assess how well the quantitative CT measurements can explain the lung function impairment, instead of testing for the optimal method. Nevertheless, we feel that air trapping measurements that combine inspiratory and expiratory CT data may be preferable when ones purpose is to quantify the individual disease components in COPD (i.e. emphysema and small airways disease). This radiologic separation of disease components may be a next step in further defining morphologic phenotypes within COPD. The solely expiratory measurement of air trapping (i.e. $\mathrm{EXP}_{-850}$ ) is not useful for this purpose, because it does not correct for air that remains in emphysematous spaces on end-expiratory CT; it thus combines quantification of emphysema and small airways disease in one measure. Therefore, we intended to eliminate emphysema from this measurement by calculating the EXP $_{-850 t o-910}$, since Gevenois et al demonstrated that voxels with a density below $-910 \mathrm{HU}$ on expiratory CT represent emphysema [22]. However, the performance of the models including this measure were worse, which makes us believe that the air trapping measurements that combine inspiration and expiration CT data will outperform the $\mathrm{EXP}_{-850 \text { to-910 }}$ when confronted with a proper reference standard.
Since only a moderate number of GOLD3 and no GOLD4 subjects were present in the screening trial, we added clinical subjects with advanced airflow limitation to our study population. Unfortunately, the inspiratory radiation exposure and the reconstruction kernel used were not identical in the clinical and screening subjects. Despite the differences, the selection of clinical subjects was reasonably the best achievable option to investigate the total spectrum of COPD. The use of two different reconstruction filters and a difference in inspiratory radiation dose may have had some influence on the quantitative CT measurements [35-38]. However, it has also been suggested that dose-reduction to as low as $20 \mathrm{mAs}$ can be applied without clinical importance [39-41]. Moreover, after analyzing only the screening trial participants, examined with a single CT system and protocol, the pattern of univariate- and multivariate regression analysis remained the same as in the total population, though was less strong after exclusion of the 74 more severely affected cases. These findings support the validity of our conclusions.

Our study has some limitations. First, there is no good reference standard for air trapping, which is an in vivo phenomenon. This limitation makes it difficult to prove that the combined inspiratory-expiratory air trapping measurements are the best methods to quantify air trapping outside emphysematous spaces. Second, CT data acquisitions were not spirometrically controlled, which would enable a standardized inspiration and expiration level. Nevertheless, its absence strengthens the generalizability of our results since spirometer-gated CT is not widely applied. Despite these limitations, the combination of CT emphysema and CT air trapping explains a large part of the lung function variability. Third, we acknowledge that spirometry was obtained before bronchodilator administration, and that some asthma, whether or not coexisting with COPD, can be present. However, this likely concerns only a small amount of heavy smoking subjects. Last, we used a retrospective design and added a separate patient group due to the paucity of severely obstructed subjects in the screening group. However, this was the best achievable option to investigate the total spectrum of COPD. Moreover, although the explained variance decreased, the pattern found did not change after exclusion of the severely affected subjects.

In summary, quantitative CT measures of emphysema and air trapping are strongly associated with airflow limitation in a population that covers the total range of airflow limitation; the combination of CT emphysema and air trapping, as simple density measurements on paired inspiratory and expiratory $\mathrm{CT}$ images, explains around three-quarters of the lung function variability in such a population of COPD subjects. We suggest that these measures might gain a role in the identification of subjects with suspected lung function impairment, based on radiological data, and might prove useful in phenotyping COPD. This may be especially interesting in lung cancer 
screening, where large numbers of examinations are obtained in heavy smokers.

Open Access This article is distributed under the terms of the Creative Commons Attribution Noncommercial License which permits any noncommercial use, distribution, and reproduction in any medium, provided the original author(s) and source are credited.

\section{Appendix 1}

To score lung segmentation the observer was able to scroll through the entire scan with a semi-translucent colour overlay of the segmentation. This overlay could be removed and replaced at any time. The guidelines for scoring, as provided to the observers, was as follows:

Excellent: over 95\% of the segmentation is correct Minor errors: $80-95 \%$ of the segmentation is correct Major errors: $50-80 \%$ of the segmentation is correct Totally wrong: less than $50 \%$ of the segmentation is correct

\section{Appendix 2}

Table 4 Results of univariate linear regression analysis for quantitative CT emphysema and air trapping measurements and pulmonary function tests in a subset of 174 subjects $^{\text {a }}$

\begin{tabular}{lcccc}
\hline & $\operatorname{logFEV}_{1}$ & $\mathrm{FEV}_{1} / \mathrm{FVC}$ & $\mathrm{RV}^{\mathrm{TTLC}}{ }^{\mathrm{b}}$ & $\mathrm{Kco}^{\mathrm{c}}$ \\
\hline CT emphysema & & & & \\
logIN_950 & $0.25^{*}$ & $0.46^{*}$ & $0.07^{*}$ & $0.25^{*}$ \\
Perc 15 & $0.14^{*}$ & $0.35^{*}$ & $0.03^{\mathrm{NS}}$ & $0.16^{*}$ \\
CT air trapping & & & & \\
EX_850 & $0.41^{*}$ & $0.54^{*}$ & $0.24^{*}$ & $0.15^{*}$ \\
EX_850to-910 $^{*}$ & $0.36^{*}$ & $0.44^{*}$ & $0.24^{*}$ & $0.11^{\dagger}$ \\
RVC $_{-860 t o-950}$ & $0.28^{*}$ & $0.21^{*}$ & $0.08^{*}$ & $0.01^{\mathrm{NS}}$ \\
E/I-ratio & $0.34^{*}$ & $0.34^{*}$ & $0.27^{*}$ & $0.09^{\dagger}$ \\
\hline
\end{tabular}

Data given are r-squared $\left(\mathrm{R}^{2}\right)$ values

${ }^{a}$ Lung cancer screening trial participants only, controls to GOLD3; ${ }^{b}$ 71 subjects with body box measurements; ${ }^{\mathrm{c}} 70$ subjects with diffusion capacity measurements

${ }^{*} p<0.001 ;{ }^{\dagger} p<0.01{ }^{\ddagger} p<0.05$; ${ }^{\mathrm{NS}}$ not significant; $\log \mathrm{IN}_{-950}=\log -$ transformed emphysema score below $-950 \mathrm{HU}$ in inspiratory scan; Perc15 = emphysema score as 15 th percentile of attenuation distribution curve in inspiratory scan; $\mathrm{EXP}_{-850}=$ air trapping score below $-850 \mathrm{HU}$ in expiratory scan; $\mathrm{EXP}_{-850 t 0-910}=$ air trapping score between $-850 \mathrm{HU}$ and $-910 \mathrm{HU}$ in expiratory scan; $\mathrm{RVC}_{-860 t o-950}=$ air trapping score as relative volume change between $-860 \mathrm{HU}$ and $950 \mathrm{HU} ; \mathrm{E} / \mathrm{I}-$ ratio $_{\mathrm{MLD}}=$ expiration to inspiration ratio of mean lung density

\section{References}

1. Global Initiative for Chronic Obstructive Pulmonary Disease. Global strategy for the diagnosis, management, and prevention of chronic obstructive pulmonary disease (2009) Available at http:// www.goldcopd.com. Accessed March 2011

2. Pauwels RA, Buist AS, Calverley PM, Jenkins CR, Hurd SS (2001) Global strategy for the diagnosis, management, and prevention of chronic obstructive pulmonary disease. NHLBI/WHO Global Initiative for Chronic Obstructive Lung Disease (GOLD) Workshop summary. Am J Respir Crit Care Med 163:1256-1276

3. Hogg JC (2004) Pathophysiology of airflow limitation in chronic obstructive pulmonary disease. Lancet 364:709-721

4. Muller NL, Staples CA, Miller RR, Abboud RT (1988) "Density mask". An objective method to quantitate emphysema using computed tomography. Chest 94:782-787

5. Nakano Y, Wong JC, de Jong PA, Buzatu L, Nagao T, Coxson HO, Elliott WM, Hogg JC, Pare PD (2005) The prediction of small airway dimensions using computed tomography. Am J Respir Crit Care Med 171:142-146

6. Matsuoka S, Kurihara Y, Yagihashi K, Hoshino M, Watanabe N, Nakajima Y (2008) Quantitative assessment of air trapping in chronic obstructive pulmonary disease using inspiratory and expiratory volumetric MDCT. AJR Am J Roentgenol 190:762769

7. Reilly JJ (2008) COPD and declining FEV1-time to divide and conquer? N Engl J Med 359:1616-1618

8. Rennard SI, Vestbo J (2008) The many "small COPDs": COPD should be an orphan disease. Chest 134:623-627

9. Han MK, Agusti A, Calverley PM, Celli BR, Criner G, Curtis JL, Fabbri LM, Goldin JG, Jones PW, MacNee W, Make BJ, Rabe KF, Rennard SI, Sciurba FC, Silverman EK, Vestbo J, Washko GR, Wouters EF, Martinez FJ (2010) Chronic obstructive pulmonary disease phenotypes: the future of COPD. Am J Respir Crit Care Med 182:598-604

10. Agusti A, Calverley PM, Celli B, Coxson HO, Edwards LD, Lomas DA, MacNee W, Miller BE, Rennard S, Silverman EK, Tal-Singer R, Wouters E, Yates JC, Vestbo J (2010) Characterisation of COPD heterogeneity in the ECLIPSE cohort. Respir Res 11:122

11. Hoop BJ, Mohamed Hoesein F, Zanen P, Gietema HA, van Ginneken B, Isgum I, Mol C, van Klaveren R, Dijkstra A, Boezen H, Groen H, Postma D, Lammers JW, Prokop M (2011) CTquantified emphysema in heavy smokers: predictive value for rate of lung function decline. Thorax. doi:10.1136/thx.2010.145995

12. van Iersel CA, de Koning HJ, Draisma G, Mali WP, Scholten ET, Nackaerts K, Prokop M, Habbema JD, Oudkerk M, van Klaveren RJ (2007) Risk-based selection from the general population in a screening trial: selection criteria, recruitment and power for the Dutch-Belgian randomised lung cancer multi-slice CT screening trial (NELSON). Int J Cancer 120:868-874

13. Quanjer PH, Tammeling GJ, Cotes JE, Pedersen OF, Peslin R, Yernault JC (1993) Lung volumes and forced ventilatory flows. Report Working Party Standardization of Lung Function Tests, European Community for Steel and Coal. Official Statement of the European Respiratory Society. Eur Respir J Suppl 16:5-40

14. Miller MR, Crapo R, Hankinson J, Brusasco V, Burgos F, Casaburi R, Coates A, Enright P, van der Grinten CP, Gustafsson P, Jensen R, Johnson DC, MacIntyre N, McKay R, Navajas D, Pedersen OF, Pellegrino R, Viegi G, Wanger J (2005) General considerations for lung function testing. Eur Respir J 26:153-161

15. van Rikxoort EM, de Hoop B, Viergever MA, Prokop M, van Ginneken B (2009) Automatic lung segmentation from thoracic computed tomography scans using a hybrid approach with error detection. Med Phys 36:2934-2947 
16. Schilham AM, van Ginneken B, Gietema H, Prokop M (2006) Local noise weighted filtering for emphysema scoring of low-dose CT images. IEEE Trans Med Imaging 25:451-463

17. Gevenois PA, De Maertelaer V, De Vuyst P, Zanen J, Yernault JC (1995) Comparison of computed density and macroscopic morphometry in pulmonary emphysema. Am J Respir Crit Care Med 152:653-657

18. Gevenois PA, De Vuyst P, De Maertelaer V, Zanen J, Jacobovitz D, Cosio MG, Yernault JC (1996) Comparison of computed density and microscopic morphometry in pulmonary emphysema. Am J Respir Crit Care Med 154:187-192

19. Newell JD Jr, Hogg JC, Snider GL (2004) Report of a workshop: quantitative computed tomography scanning in longitudinal studies of emphysema. Eur Respir J 23:769-775

20. van Ginneken B, Murphy K, van Rikxoort EM, Isgum I, de Hoop B, Prokop M, de Jong PA, Gietema HA (2009) Quantification of Emphysema and Small Airway Disease in COPD Patients from Lobar Analysis of Volumetric Inspiration and Expiration Thoracic CT Scans. Radiological Society of North America, 95th Annual Meeting

21. Busacker A, Newell JD Jr, Keefe T, Hoffman EA, Granroth JC, Castro M, Fain S, Wenzel S (2009) A multivariate analysis of risk factors for the air-trapping asthmatic phenotype as measured by quantitative $\mathrm{CT}$ analysis. Chest 135:48-56

22. Gevenois PA, De Vuyst P, Sy M, Scillia P, Chaminade L, de Maertelaer V, Zanen J, Yernault JC (1996) Pulmonary emphysema: quantitative CT during expiration. Radiology 199:825-829

23. Kubo K, Eda S, Yamamoto H, Fujimoto K, Matsuzawa Y, Maruyama Y, Hasegawa M, Sone S, Sakai F (1999) Expiratory and inspiratory chest computed tomography and pulmonary function tests in cigarette smokers. Eur Respir J 13:252-256

24. Eda S, Kubo K, Fujimoto K, Matsuzawa Y, Sekiguchi M, Sakai F (1997) The relations between expiratory chest CT using helical $\mathrm{CT}$ and pulmonary function tests in emphysema. Am J Respir Crit Care Med 155:1290-1294

25. O'Donnell RA, Peebles C, Ward JA, Daraker A, Angco G, Broberg P, Pierrou S, Lund J, Holgate ST, Davies DE, Delany DJ, Wilson SJ, Djukanovic R (2004) Relationship between peripheral airway dysfunction, airway obstruction, and neutrophilic inflammation in COPD. Thorax 59:837-842

26. Goedhart DM, Zanen P (2002) Selecting the best method to evaluate bronchodilation when analysing bronchodilator studies. Stat Med 21:3677-3685

27. Fox J (1991) Regression diagnostics. Sage Publications, Thousand Oak

28. Nakano Y, Muro S, Sakai H, Hirai T, Chin K, Tsukino M, Nishimura K, Itoh H, Pare PD, Hogg JC, Mishima M (2000) Computed tomographic measurements of airway dimensions and emphysema in smokers. Correlation with lung function. Am J Respir Crit Care Med 162:1102-1108

29. Achenbach T, Weinheimer O, Biedermann A, Schmitt S, Freudenstein D, Goutham E, Kunz RP, Buhl R, Dueber C, Heussel CP (2008) MDCT assessment of airway wall thickness in COPD patients using a new method: correlations with pulmonary function tests. Eur Radiol $18: 2731-2738$
30. Hasegawa M, Nasuhara Y, Onodera Y, Makita H, Nagai K, Fuke S, Ito Y, Betsuyaku T, Nishimura M (2006) Airflow limitation and airway dimensions in chronic obstructive pulmonary disease. Am J Respir Crit Care Med 173:1309-1315

31. Matsuoka S, Kurihara Y, Yagihashi K, Hoshino M, Nakajima Y (2008) Airway dimensions at inspiratory and expiratory multisection CT in chronic obstructive pulmonary disease: correlation with airflow limitation. Radiology 248:1042-1049

32. Yamashiro T, Matsuoka S, Estepar RS, Dransfield MT, Diaz A, Reilly JJ, Patz S, Murayama S, Silverman EK, Hatabu H, Washko GR, Quantitative assessment of bronchial wall attenuation with thin-section CT (2010) An indicator of airflow limitation in chronic obstructive pulmonary disease. AJR Am J Roentgenol 19:363-369

33. Lee YK, Oh YM, Lee JH, Kim EK, Lee JH, Kim N, Seo JB, Lee SD (2008) Quantitative assessment of emphysema, air trapping, and airway thickening on computed tomography. Lung 186:157165

34. Yamashiro T, Matsuoka S, Bartholmai BJ, San Jose ER, Ross JC, Diaz A, Murayama S, Silverman EK, Hatabu H, Washko GR (2010) Collapsibility of lung volume by paired inspiratory and expiratory CT scans: correlations with lung function and mean lung density. Acad Radiol 17:489-495

35. Gierada DS, Bierhals AJ, Choong CK, Bartel ST, Ritter JH, Das NA, Hong C, Pilgram TK, Bae KT, Whiting BR, Woods JC, Hogg JC, Lutey BA, Battafarano RJ, Cooper JD, Meyers BF, Patterson GA (2010) Effects of CT section thickness and reconstruction kernel on emphysema quantification relationship to the magnitude of the CT emphysema index. Acad Radiol 17:146-156

36. Ley-Zaporozhan J, Ley S, Weinheimer O, Iliyushenko S, Erdugan S, Eberhardt R, Fuxa A, Mews J, Kauczor HU (2008) Quantitative analysis of emphysema in 3D using MDCT: influence of different reconstruction algorithms. Eur J Radiol 65:228-234

37. Boedeker KL, Nitt-Gray MF, Rogers SR, Truong DA, Brown MS, Gjertson DW, Goldin JG (2004) Emphysema: effect of reconstruction algorithm on CT imaging measures. Radiology 232:295301

38. Yuan R, Mayo JR, Hogg JC, Pare PD, McWilliams AM, Lam S, Coxson HO (2007) The effects of radiation dose and CT manufacturer on measurements of lung densitometry. Chest 132:617-623

39. Madani A, De Maertelaer V, Zanen J, Gevenois PA (2007) Pulmonary emphysema: radiation dose and section thickness at multidetector CT quantification-comparison with macroscopic and microscopic morphometry. Radiology 243:250-257

40. Gierada DS, Pilgram TK, Whiting BR, Hong C, Bierhals AJ, Kim $\mathrm{JH}$, Bae KT (2007) Comparison of standard- and low-radiationdose CT for quantification of emphysema. AJR Am J Roentgenol 188:42-47

41. Zaporozhan J, Ley S, Weinheimer O, Eberhardt R, Tsakiris I, Noshi Y, Herth F, Kauczor HU (2006) Multi-detector CT of the chest: influence of dose onto quantitative evaluation of severe emphysema: a simulation study. J Comput Assist Tomogr 30:460 468 